\title{
Supporting Information for: Enrichment Tags for Enhanced-Resolution Profiling of the Polar Metabolome
}

\author{
Erin E. Carlson and Benjamin F. Cravatt
}

The Skaggs Institute for Chemical Biology and Department of Chemical Physiology, The Scripps Research Institute, 10550 North Torrey Pines Road, La Jolla, CA 92037

\section{Supporting Information Contents:}

\section{Methods:}

General procedures and materials

Synthesis and characterization of p-chlorophenylalanine-containing peptides

Protocol for acquisition of accurate and tandem MS data

Supporting Information Table 1. Table of identified metabolites.

Supporting Information Figure 1. Expansion of the polar region of the data comparing the retention and separation of small molecules conjugated to either the original or Cl-Phe acid-capture tags (expansion of Figure 2).

Supporting Information Figure 2. Comparison of the LC elution profiles of small-molecule standards conjugated to the original tag and resolved under the original LC elution protocol (A, derived from ref. 1) or the modified LC elution protocol used for Cl-Phe tags (B).

Supporting Information Figure 3. $\mathrm{MS}^{2}$ analysis of endogenous metabolites and synthetic standards with the amine-capture tags.

Supporting Information Figure 4. $\mathrm{MS}^{2}$ analysis of endogenous metabolites and synthetic standards with the acid-capture tags.

Supporting Information Figure 5. $\mathrm{MS}^{2}$ analysis of endogenous metabolites and synthetic standards with the thiol-capture tags.

Supporting Information Figure 6. $\mathrm{MS}^{2}$ analysis of endogenous metabolites and synthetic standards with the ketone/aldehyde-capture tags. 
Supporting Information Figure 7. Theoretical and experimental isotopic abundance patterns for singlyand multiply-labeled succinic acid. 


\section{Methods}

General procedures and materials. All resin reactions were performed in fritted vessels (Biorad). Presence of a free amine was determined by the Kaiser ninhydrin test for amines. Sequence grade modified trypsin was purchased from Promega (Madison, WI). PEGA amino resin and all amino acids were purchased from EMD Biosciences. Sample analysis was performed on an Agilent 1100 LC-MSD using a reverse phase column (Phenomenex Luna $\mathrm{C}_{18}, 5 \mu \mathrm{m}, 150$ x $4.6 \mathrm{~mm}$ ) detected by electrospray ionization (positive ion mode). With the original tags, analysis was initiated with an isocratic elution of $100 \% \mathrm{~A}$ at $0.25 \mathrm{~mL} / \mathrm{min}$ for 5 min followed by a linear gradient of $0-100 \% \mathrm{~B}$ at $0.5 \mathrm{~mL} / \mathrm{min}$ over $35 \mathrm{~min}$, then an isocratic elution at 100\% B for 9 min and re-equilibration with 100\% A for 4 min (A: 95:5 $\mathrm{H}_{2} \mathrm{O}: \mathrm{CH}_{3} \mathrm{CN}, 0.1 \%$ formic acid; B: 95:5 $\mathrm{CH}_{3} \mathrm{CN}: \mathrm{H}_{2} \mathrm{O}, 0.1 \%$ formic acid). With the Cl-Phe-functionalized tags, analysis was initiated with an isocratic elution of $100 \% \mathrm{~A}$ at $0.25 \mathrm{~mL} / \mathrm{min}$ for $2 \mathrm{~min}$ followed by a the following step gradients (all at 0.5 mL/min): 0-10\% B over 3 min, 10-15\% B over 5 min, 15-35\% B over 15 min, 35-75\% B over 10 min, 75$100 \%$ B over 5 min, then an isocratic elution at 100\% B for 5 min and re-equilibration with $100 \%$ A for 4 min (A: 95:5 $\mathrm{H}_{2} \mathrm{O}: \mathrm{CH}_{3} \mathrm{CN}, 0.1 \%$ formic acid; B: 95:5 $\mathrm{CH}_{3} \mathrm{CN}: \mathrm{H}_{2} \mathrm{O}, 0.1 \%$ formic acid). Under these modified LC-elution conditions, metabolites conjugate to the original tag still showed poor retention and resolution (Supporting Information Figure 2). Accurate mass determinations and MS/MS experiments were performed in the positive-ion mode using an LTQ-Orbitrap Hybrid mass spectrometer (Thermo Electron, Bremen, Germany) equipped with an Agilent 1100 LC using a reverse phase column (Phenomenex Luna $\mathrm{C}_{18}$, $5 \mu \mathrm{m}, 150 \times 4.6 \mathrm{~mm}$, gradients as above) and Thermo Electron ESI source. Metabolite identifies that could not be confirmed with MS/MS data were evaluated with co-elution studies. ${ }^{1} \mathrm{H}$ and ${ }^{13} \mathrm{C}$ nuclear magnetic resonance (NMR) spectra were recorded on a Bruker DRX-600 and chemical shifts reported relative to residual solvent peaks in parts per million. Peak multiplicity is reported as: singlet (s), doublet (d), triplet (t), and multiplet (m). Procedures for synthesis of the original tags and culture of MDA-MB-231 cells were performed as described previously. ${ }^{1}$ 


\section{Functionalization of PEGA resin.}

Carboxylic acid capture resin: The lysine-containing peptide (1.4 equiv), HBTU (1.4 equiv), HOBt (1.3 equiv), and DIPEA (3.4 equiv) were dissolved in DMF (0.1 M), added to PEGA amino resin (washed 3x with DMF), and agitated overnight. Resin was deprotected just prior to use with a $4 \%$ solution of hydrazine hydrate in DMF (5 x 3 min) to provide the carboxylic acid capture resin.

Ketone and aldehyde capture resin: The above prepared acid capture resin was functionalized with $\mathrm{Boc}_{2}$ amino-oxyacetic acid by dissolution of this compound (1.4 equiv) along with HBTU (1.4 equiv), HOBt (1.3 equiv), and DIPEA (2 equiv) in DMF (0.1 M) followed by addition of this solution to the resin and agitation overnight. Resin was deprotected just prior to use with TFA:SiEt ${ }_{3} \mathrm{H}: \mathrm{CH}_{2} \mathrm{Cl}_{2}(95: 2.5: 2.5)$ under a nitrogen atmosphere to minimize reaction with ambient acetone to provide the ketone and aldehyde capture resin. Thiol capture resin: The above prepared acid capture resin was functionalized with 4-maleimidobutyric acid by dissolution of this compound (3 equiv) along with HBTU (3 equiv), HOBt (2.9 equiv), and DIPEA (6 equiv) in DMF (0.1 M) followed by addition of this solution to the resin and agitation for $4 \mathrm{~h}$.

Amine capture resin: The glutamate-containing peptide (1.4 equiv), HBTU (1.4 equiv), HOBt (1.3 equiv) and DIPEA (3.4 equiv) were dissolved in DMF (0.1 M), added to PEGA amine resin (washed 3x with DMF), and agitated overnight. Resin was washed with above described protocol. Resin was deprotected prior to use with a solution of $\mathrm{Pd}\left(\mathrm{Ph}_{3} \mathrm{P}\right)_{4}\left(0.05\right.$ equiv) and phenylsilane (25 equiv) in $\mathrm{CH}_{2} \mathrm{Cl}_{2}$ (3 $\left.\mathrm{x} 5 \mathrm{~min}\right)$ to provide the amine capture resin.

Metabolome preparation for METPR analysis. Homogenized cell preparations [ 1 x $10^{7}$ cells per experiment homogenized by sonication (0.3 s, 10 pulses) in phosphate buffer, $\mathrm{pH} 8.0(100 \mu \mathrm{L})]$ were treated with $\mathrm{CH}_{3} \mathrm{CN}(200 \mu \mathrm{L})$, vortexed, and allowed to stand on ice for $10 \mathrm{~min}$. The sample was then centrifuged at 13,200 x $g$ for 10 min. Next, DMF (900 $\mu \mathrm{L}$ ) was added, the sample vortexed, and centrifuged immediately. 
The supernatant was transferred to a test tube and evaporated under a stream of nitrogen ( 10 min) to remove the $\mathrm{CH}_{3} \mathrm{CN}$. The resulting metabolite solution was added to capture resin as detailed below.

General procedures for reaction of metabolomes with resins.

Amine capture resin: $30 \mathrm{mg}$ of resin (per $10^{7}$ cells) was pre-activated as the succinimidyl ester by reaction with $O$-(7-azabenzotriazol-1-yl)- $N, N, N^{\prime}, N^{\prime}$-tetramethyluronium hexafluorophosphate (HATU, 3 eq.), $N$ hydroxysuccinimide (NHS, 3 eq.), and $N, N$-diisopropylethylamine (DIPEA, 3 eq.) in DMF for 4 h. The resin was rinsed with DMF (3x) and subjected to the metabolite solution.

Acid capture resin: HBTU (2 eq.) and 1-hydroxybenzotriazole hydrate (HOBt, 2 eq.) were added to the small molecule mixture and allowed to stand for $1 \mathrm{~min}$. The mixture was then added to the aminefunctionalized resin (23 mg).

Ketone/aldehyde capture resin: The small molecule mixture was added directly to alkoxyl amine functionalized-resin (30 mg). Capture was performed under nitrogen to minimize reaction of the resin with ambient acetone.

Thiol capture resin: The small molecule mixture was added directly to maleimide-functionalized resin (23 mg).

All reactions were rotated overnight at $25{ }^{\circ} \mathrm{C}$, and the resins were then washed with $\mathrm{DMF}, \mathrm{CH}_{2} \mathrm{Cl}_{2}, \mathrm{CH}_{2} \mathrm{Cl}_{2}$ : $\mathrm{CH}_{3} \mathrm{OH}$ (1:1), hexanes, $\mathrm{CH}_{3} \mathrm{OH}, \mathrm{H}_{2} \mathrm{O}$, and $\mathrm{PBS}$ (all 3x, $10 \mathrm{~mL}$ ). Trypsinization was initiated by addition of 4 $\mu$ g sequence grade modified trypsin (Promega) in $\mathrm{PBS}$ (pH 7.4), 5 mM CaCl $2,0.05 \% \mathrm{AcOH}_{\text {(105 }}$ $\mu \mathrm{L} /$ reaction). Cleavage was carried out at $37^{\circ} \mathrm{C}$ for $2 \times 3$ h with shaking. Trypsin-released molecules were analyzed on an Agilent 1100 LC-MSD using a reverse phase column with the gradients described above (Phenomenex Luna $\mathrm{C}_{18}, 5 \mu \mathrm{m}, 150 \times 4.6 \mathrm{~mm}$ ) and detected by electrospray ionization (positive ion mode). 
Synthesis of Succinyl-Arg-Lys-Cl-Phe- $\mathrm{NH}_{2}$. Rink amide MBHA resin (1.0 g, $0.58 \mathrm{mmol}$ ) was added to a fritted vessel and deprotected with $20 \%$ piperidine in DMF (8 mL total volume) for 20 min (vessel was capped and gently agitated on a tube rotator). Resin was washed with the following sequence of solvents three times: DMF (3x) and $\mathrm{CH}_{2} \mathrm{Cl}_{2}$ (3x). Fmoc-Cl-Phe-OH (390 mg, 0.94 mmol, 1.3 equiv), O-(benzotriazol1-yl)- $N, N, N^{\prime} N^{\prime}$-tetramethyluronium hexafluorophosphate (HBTU) (360 mg, 0.94 mmol, 1.3 equiv), and 1hydroxybenzotriazole hydrate (HOBt) (120 mg, $0.86 \mathrm{mmol}, 1.2$ equiv) were dissolved in DMF (8 mL), to which $N, N$-diisopropylethylamine (DIPEA) (250 $\mu \mathrm{L}, 1.4 \mathrm{mmol}, 2$ equiv) was added. After $10 \mathrm{~min}$, this solution was added to the deprotected resin, the vessel was capped and agitated overnight. Resin was washed using the above procedure, deprotected, and again washed. Fmoc-Lys(ivDde)-OH (2.0 g, 3.9 mmol, 4 equiv), HBTU (1.3 g, 3.9 mmol, 4 equiv) and HOBt (520 mg, 3.4 mmol, 3.9 equiv) were dissolved in DMF (8 mL), to which DIPEA (1.2 mL, $7.0 \mathrm{mmol}, 8$ equiv) was added. After $10 \mathrm{~min}$, this solution was added to the ClPhe-functionalized resin, the vessel was capped and agitated overnight. Resin was washed, deprotected and again washed. Next, Fmoc-Arg(Pbf)-OH (1.5 g, 2.3 mmol, 4 equiv) and O-(7-Azabenzotriazol-1-yl)$N, N, N^{\prime}, N^{\prime}$-tetramethyluronium hexafluorophosphate (HATU) (880 mg, 2.3 mmol, 4 equiv) were dissolved in DMF (8 mL) to which DIPEA (800 $\mu \mathrm{L}, 4.6 \mathrm{mmol}, 8$ equiv) was added and allowed to stand for $10 \mathrm{~min}$. This solution was added to the resin and the vessel was capped and agitated overnight. Resin was washed, deprotected, and again washed. The resin bound tripeptide was then suspended in $\mathrm{CH}_{2} \mathrm{Cl}_{2}(8 \mathrm{~mL})$, pyridine (580 $\mu \mathrm{L}, 7.0 \mathrm{mmol}, 12$ equiv) and succinic anhydride (560 mg, $5.8 \mathrm{mmol}, 10$ equiv) added, and the resin agitated overnight. After reaction completion, the resin was cleaved with $5 \mathrm{~mL}$ of a TFA:SiEt $\mathrm{H}_{2} \mathrm{CH}_{2} \mathrm{Cl}_{2}$ (95:2.5:2.5) solution for $1 \mathrm{~h}$. The resin was rinsed with additional TFA and $\mathrm{CH}_{2} \mathrm{Cl}_{2}$. Solvent was removed under a stream of nitrogen to give the crude product. The peptide was precipitated from ether (2x) and dried under high vacuum to give an average yield of $260 \mathrm{mg}$ of the TFA salt (72\% yield). ${ }^{1} \mathrm{H}$ NMR (600 MHz, $\mathrm{d}_{6}{ }^{-}$ DMSO) $\delta 8.37$ (d, $1 \mathrm{H}, J=7.2 \mathrm{~Hz}), 8.18$ (d, $1 \mathrm{H}, J=7.8 \mathrm{~Hz}), 8.09$ (d, $1 \mathrm{H}, J=8.4 \mathrm{~Hz}), 7.80$ (t, $1 \mathrm{H}, J=6.0$ Hz), 7.63 (s, $1 \mathrm{H}), 7.53$ (d, 2 H, $J=8.4 \mathrm{~Hz}$ ), 7.47 (d, 2 H, $J=8.4$ Hz), 7.37 (s, $1 \mathrm{H}), 4.63-4.67$ (m, $1 \mathrm{H}), 4.46-$ 4.49 (m, 1 H), 4.37-4.41 (m, 1 H), 3.61-3.66 (m, 2 H), 3.29-3.34 (m, 2 H), 3.21-3.26 (m, 3 H), 3.03-3.07 (m, 
1 H), 2.73-2.74 (m, 1 H), 2.60-2.69 (m, 4 H), 2.04-2.09 (m, 1 H), 1.69-1.92 (m, 8 H), 1.46-1.54 (m, 2 H), 1.17 (s, $6 \mathrm{H}), 1.15$ (d, $6 \mathrm{H}, J=7.2 \mathrm{~Hz}$ ); ${ }^{13} \mathrm{C}$ NMR (150 MHz, d $\mathrm{d}_{6}$-DMSO) $\delta 196.5,175.3,173.9,172.6,171.6$, 171.6, 171.2, 162.4, 158.7 (q, $J=36 \mathrm{~Hz}$ (TFA)), 156.8, 136.8, 131.1, 128.0, 115.8 (q, $J=290 \mathrm{~Hz}(\mathrm{TFA}))$, 106.3, 65.0, 53.5, 52.7, 52.5, 52.4, 43.0, 40.5, 10.0, 36.9, 36.3, 31.2, 30.0, 29.6, 29.2, 28.9, 28.6, 28.4, 27.8, 25.0, 22.6, 22.2, 6.7, 6.0. ESI-Orbitrap (m/z) $[\mathrm{M}+\mathrm{H}]$ calcd for $\mathrm{C}_{38} \mathrm{H}_{58} \mathrm{~N}_{8} \mathrm{O}_{8} \mathrm{Cl}$ 789.40607, found 789.40598.

Synthesis of Succinyl-Arg-Glu(Cl-Phe- $\left.\mathrm{NH}_{2}\right)$-OAll. Rink amide MBHA resin (1.0 g, 0.58 mmol) was added to a fritted vessel and deprotected with $20 \%$ piperidine in DMF (8 mL total volume) for 20 min (vessel was capped and gently agitated on a tube rotator). Resin was washed with the following sequence of solvents three times: DMF (3x) and $\mathrm{CH}_{2} \mathrm{Cl}_{2}$ (3x). Fmoc-Cl-Phe-OH (390 mg, 0.94 mmol, 1.3 equiv), HBTU (360 mg, $0.94 \mathrm{mmol}, 1.3$ equiv), and HOBt (120 mg, $0.86 \mathrm{mmol}, 1.2$ equiv) were dissolved in DMF (8 mL), to which DIPEA (250 $\mu \mathrm{L}, 1.4 \mathrm{mmol}, 2$ equiv) was added. After $10 \mathrm{~min}$, this solution was added to the deprotected resin, the vessel was capped and agitated overnight. Resin was washed using the above procedure, deprotected, and again washed. Fmoc-Glu-OAll (480 mg, 1.2 mmol, 2 equiv), HBTU (450 mg, 1.2 mmol, 2 equiv), and HOBt (170 mg, $1.1 \mathrm{mmol}, 1.9$ equiv) were dissolved in DMF (8 mL) to which DIPEA (400 $\mu \mathrm{L}$, $2.3 \mathrm{mmol}, 4$ equiv) was added. After $10 \mathrm{~min}$, this solution was added to the Cl-Phe-functionalized resin, the vessel was capped and agitated overnight. Resin was washed, deprotected and again washed. Next, FmocArg(Pbf)-OH (1.5 g, 2.3 mmol, 4 equiv) and HATU (880 mg, 2.3 mmol, 4 equiv) were dissolved in DMF (8 $\mathrm{mL}$ ) to which DIPEA (800 $\mu \mathrm{L}, 4.6 \mathrm{mmol}, 8$ equiv) was added and allowed to stand for $10 \mathrm{~min}$. This solution was added to the resin and the vessel was capped and agitated overnight. Resin was washed, deprotected, and again washed. The resin bound tripeptide was then suspended in $\mathrm{CH}_{2} \mathrm{Cl}_{2}(8 \mathrm{~mL})$, pyridine (580 $\mu \mathrm{L}, 7.0$ mmol, 12 equiv) and succinic anhydride (560 mg, $5.8 \mathrm{mmol}, 10$ equiv) added, and the resin agitated overnight. After reaction completion, the resin was cleaved with $5 \mathrm{~mL}$ of a TFA:SiEt $\mathrm{H}_{3} \mathrm{CH}_{2} \mathrm{Cl}_{2}$ (95:2.5:2.5) solution for $1 \mathrm{~h}$. The resin was rinsed with additional TFA and $\mathrm{CH}_{2} \mathrm{Cl}_{2}$. Solvent was removed under a stream of nitrogen to give the crude product. The peptide was precipitated from ether $(2 \mathrm{x})$ and dried under high 
vacuum to give an average yield of $190 \mathrm{mg}$ of the TFA salt (64\% yield). ${ }^{1} \mathrm{H}$ NMR (600 MHz, $\mathrm{d}_{6}$-DMSO) $\delta$ 8.35 (d, $1 \mathrm{H}, J=7.2 \mathrm{~Hz}$ ), 8.05 (d, $1 \mathrm{H}, J=7.8 \mathrm{~Hz}$ ), 7.99 (d, $1 \mathrm{H}, J=8.4 \mathrm{~Hz}$ ), 7.53 (t, $1 \mathrm{H}, J=6.0 \mathrm{~Hz}$ ), 7.47 (s, 1 H), 7.30 (d, 2 H, $J$ = 8.4 Hz), 7.23 (d, 2 H, $J=8.4$ Hz), 7.08 (s, 1 H), 5.85-5.91 (m, 1 H), 5.29-5.32 (m, 1 H), 5.20-5.22 (m, 1 H), 4.55 (d, 2 H, $J=4.8$ Hz), 4.39-4.43 (m, 1 H), 4.30-4.33 (m, 1 H), 4.16-4.4.19 (m, 1 H), 3.05-3.3.11 (m, 2 H), 2.97 (dd, 1 H, $J$ = 13.8, 4.8 Hz), 2.70-2.74 (m, 1 H), 2.35-2.45 (m, 4 H), 2.12-2.19 (m, 2 H), 1.84-1.89 (m, 1 H), 1.64-1.76 (m, 2 H), 1.45-1.51 (m, 3 H); ${ }^{13} \mathrm{C}$ NMR (150 MHz, d6 - DMSO) $\delta$ 173.9, 172.9, 171.8, 171.3, 171.1, 171.0, 158.4 (q, $J=36$ Hz (TFA)), 156.7, 137.1, 132.3, 131.0, 130.9, 128.0, 117.8, 115.8 (q, $J=290 \mathrm{~Hz}(\mathrm{TFA})$ ), 64.9, 53.6, 51.8, 51.7, 40.5, 40.1, 37.0, 31.5, 29.9, 29.4, 29.1, 26.6, 24.9. ESI-Orbitrap $(\mathrm{m} / \mathrm{z})[\mathrm{M}+\mathrm{H}]$ calcd for $\mathrm{C}_{27} \mathrm{H}_{39} \mathrm{~N}_{7} \mathrm{O}_{8} \mathrm{Cl}$ 624.25432, found 624.25402.

\section{Accurate and tandem MS experiments.}

Accurate mass and $\mathrm{MS}^{2}$ experiments were performed in the positive-ion mode using an LTQ-Orbitrap Hybrid mass spectrometer (Thermo Electron, Bremen, Germany) equipped with a Thermo Electron ESI source and an Agilent 1100 LC using a reverse phase column (Phenomenex Luna $\mathrm{C}_{18}, 5 \mu \mathrm{m}, 150$ x $4.6 \mathrm{~mm}$, gradients as above). All experiments were performed using external calibration with the standard LTQ calibration mixture as per manufacturer protocols (caffeine, MRFA peptide, and Ultramark 1600). Data was acquired with the following settings: electrospray voltage $=2.0 \mathrm{kV}$, sheath gas flow rate $=30$ units, capillary temperature $=275^{\circ} \mathrm{C}$, single microscan $=1 \mathrm{~s}$, resolving power of the Ortibrap mass analyzer $=60,000$ (profile mode). For MS², precursor ions were activated using Pulsed-Q Dissociation (PQD), which enables detection of fragments with $\mathrm{m} / \mathrm{z}$ values as low as $50 .^{2}$ 
Supporting Information Table 1. Table of identified metabolites.

\begin{tabular}{|c|c|c|c|c|c|c|c|}
\hline Metabolite & $\begin{array}{c}t_{R} \\
(\min )\end{array}$ & $\begin{array}{c}\text { Peak } \\
\text { Area } \\
\left(\mathrm{x} 10^{4}\right)\end{array}$ & $\begin{array}{l}\text { Predicted } \\
\text { Mass } \\
\text { (-Tag) }\end{array}$ & $\begin{array}{l}\text { Exact Mass } \\
\text { Calculated }\end{array}$ & $\begin{array}{c}\text { Exact Mass } \\
\text { Measured }\end{array}$ & ppm & Formula \\
\hline \multicolumn{8}{|c|}{$\begin{array}{l}\text { Imine (Original } \\
\text { Tag) }\end{array}$} \\
\hline Glycine & 6.62 & 110 & 75.0 & 303.16630 & 303.16642 & 0.41 & C12H23N4O5 \\
\hline Valine & 14.22 & 99.1 & 117.1 & 345.21325 & 345.21314 & 0.31 & C15H29N4O5 \\
\hline Threonine & 6.63 & 8.37 & 119.1 & 347.19251 & 347.19232 & 0.55 & C14H27N4O6 \\
\hline Taurine & 6.64 & 21.4 & 125.0 & 353.14893 & 353.14847 & 1.31 & C12H25N4O6S \\
\hline Isoleucine & 15.90 & 78.2 & 131.1 & 359.22890 & 359.22813 & 2.14 & C16H31N4O5 \\
\hline Leucine & 16.38 & 80.7 & 131.1 & 359.22890 & 359.22829 & 1.69 & C16H31N4O5 \\
\hline Glutamine & 6.01 & 24.7 & 146.1 & 374.20341 & 374.20395 & 1.44 & C15H28N5O6 \\
\hline Glutamate & 6.63 & 157 & 147.1 & 375.18743 & 375.18679 & 1.70 & C15H27N4O7 \\
\hline Arginine & 6.25 & 98.5 & 174.1 & 402.24594 & 402.24545 & 1.23 & C16H32N7O5 \\
\hline
\end{tabular}

Amine (Cl-Phe

Tag)

Glycine

Valine

15.81

1850

75.0

385.12732

385.12697

0.92

C16H22ClN4O5

Threonine

15.70

94.4

427.17427

427.17398

0.69

C19H28CIN4O5

Taurine

17.86

119.1

429.15354

429.15320

0.00

C18H26CIN4O6

Isoleucine

22.56

2780

435.10996

435.10927

1.59

C16H24CIN4O6S

Leucine

22.93

131.1

441.18992

441.18948

1.01

C20H30CIN4O5

Glutamine

2970

131.1

441.18992

441.18967

0.58

146.1

456.16444

Glutamate

16.58

7360

456.16391

1.16

457.14845

Arginine

$12.16 \quad 16.5$

174.1

484.20697

$457.14740 \quad 2.31$

$484.20596 \quad 2.09$

C20H30ClN4O5

C19H27ClN5O6

C19H26ClN4O7

C20H31CIN7O5

Acid (Original

$$
\text { Tag) }
$$

Lactic Acid

Succinic Acid

Pyroglutamate*

Phenylalanine

Pantothenic Acid

$\begin{array}{lccc}5.68 & 384 & 90.0 & 218.14992 \\ 6.85 & 56.2 & 118.0 & 246.14483 \\ 5.90 & 644 & 129.1 & 257.16082 \\ 6.84 & 49.8 & 165.1 & 293.19720 \\ 6.85 & 10.2 & 219.1 & 347.22890\end{array}$

218.14927

2.97

C9H20N3O3

246.14506

0.92

C10H20N3O4

257.16061

0.81

C11H21N4O3

$293.19734 \quad 0.47$

C15H25N4O2

$347.22821 \quad 1.98$

C15H31N4O5

Acid (Cl-Phe

$$
\text { Tag) }
$$

\section{Lactic Acid}

$$
14.71
$$$$
7060
$$

90.0

399.17936

399.17947

0.28

C18H28CIN4O4

Succinic Acid

15.49

118.0

427.17427

427.17391

0.85

C19H28CIN4O5

Pyroglutamate*

14.08

$8680 \quad 129.1$

438.18921

2.39

C20H29CIN5O4

Phenylalanine

13.79

165.1

474.22664

474.22592

1.53

C24H33CIN5O3

$129 \quad 219.1$

528.25834

$528.25720 \quad 2.16$

C24H39CIN5O6

Thiol (Original

$$
\text { Tag) }
$$

Cysteine
Cys-Gly
Glutathione
Thiol (Cl-Phe
Tag)

$\begin{array}{llll}6.69 & 9.13 & 121.0 & 432.19113 \\ 6.93 & 15.1 & 178.0 & 489.21259 \\ 7.07 & 175 & 307.1 & 618.25519\end{array}$

$\begin{array}{ll}432.19071 & 0.98 \\ 489.21178 & 1.67 \\ 618.25400 & 1.92\end{array}$

C17H30N5O6S

C19H33N6O7S

C24H40N7O10S 


\begin{tabular}{|c|c|c|c|c|c|c|c|}
\hline Cysteine & 14.17 & 354 & 121.0 & 613.22057 & 613.21985 & 1.18 & C26H38ClN6O7S \\
\hline Cys-Gly & 12.77 & 70.8 & 178.0 & 670.24204 & 670.24113 & 1.35 & C28H41ClN7O8S \\
\hline Glutathione & 14.87 & 1090 & 307.1 & 799.28463 & 799.28386 & 0.97 & C33H48CIN8O11S \\
\hline \multicolumn{8}{|c|}{$\begin{array}{c}\text { Ket/Ald } \\
\text { (Original Tag) }\end{array}$} \\
\hline Pyruvate & 6.64 & 790 & 88.0 & 289.15065 & 289.15074 & 0.32 & C11H21N4O5 \\
\hline \multicolumn{8}{|c|}{$\begin{array}{l}\text { Ket/Ald (Cl-Phe } \\
\text { Tag) }\end{array}$} \\
\hline Pyruvate & 18.74 & 668 & 88.0 & 470.18009 & 470.17906 & 2.19 & C20H29CIN5O6 \\
\hline
\end{tabular}



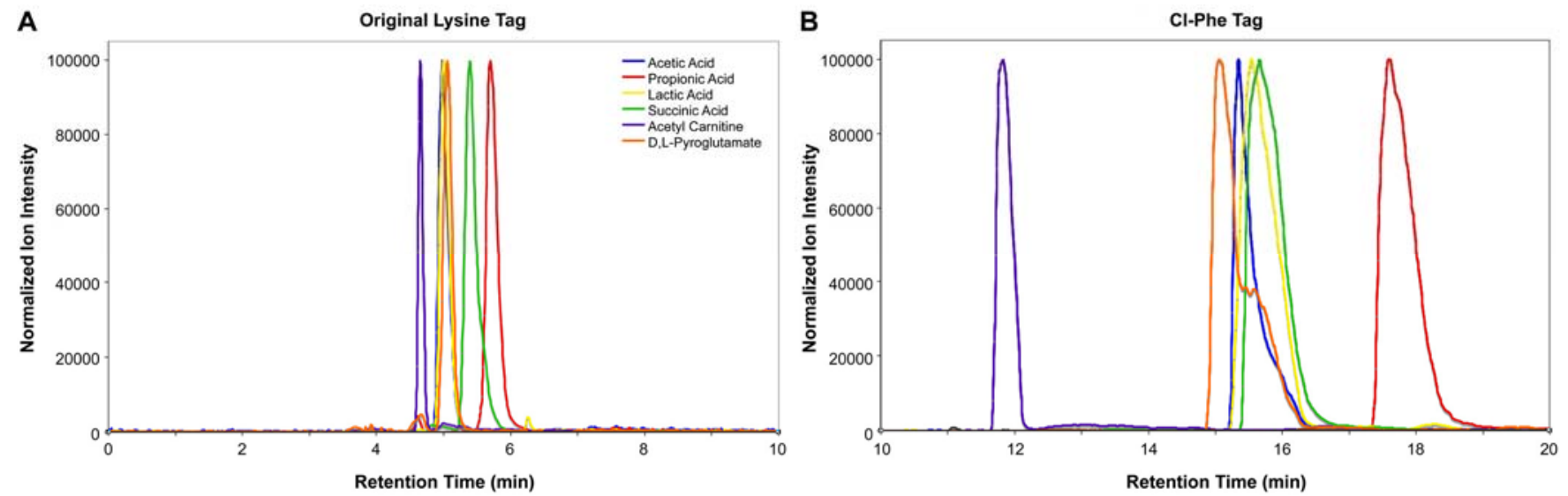

Supporting Information Figure 1. Expansion of the polar region of the data comparing the retention and separation of small molecules conjugated to either the original (A) or Cl-Phe (B) acid-capture tags (expansion of Figure 2). The broad peak shape observed for Cl-Phe-tagged D,L-pyroglutamate (orange) results from resolution of the diasteriomeric products. Original Tag Elution Times: Acetyl carnitine $t_{R}=4.66$ $\min$, Acetic acid $t_{R}=4.98 \min$, Lactic acid $t_{R}=5.00 \min , \mathrm{D}, \mathrm{L}-\mathrm{Pyroglutamate} t_{\mathrm{R}}=5.06 \mathrm{~min}$, Succinic acid $t_{R}$ $=5.39$ min, Propionic acid $t_{R}=5.70$ min. Cl-Phe Tag Elution Times: Acetyl carnitine $t_{R}=11.83$ min, Acetic acid $t_{R}=15.34 \min$, Lactic acid $t_{R}=15.54 \min , \mathrm{D}$,L-Pyroglutamate $t_{R}=15.05$ min, Succinic acid $t_{R}=15.66$ $\min$, Propionic acid $t_{R}=17.60 \mathrm{~min}$. (C) Standards tagged with the original acid-capture tag and eluted using the new gradient utilized for compounds conjugated to the Cl-Phe tag. Elution Times: Acetyl carnitine $t_{R}=$

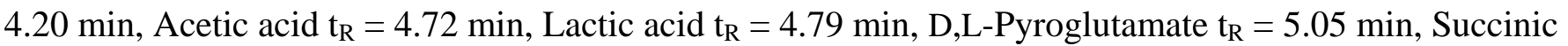
acid $t_{\mathrm{R}}=5.51 \mathrm{~min}$, Propionic acid $\mathrm{t}_{\mathrm{R}}=5.89 \mathrm{~min}$. 
A

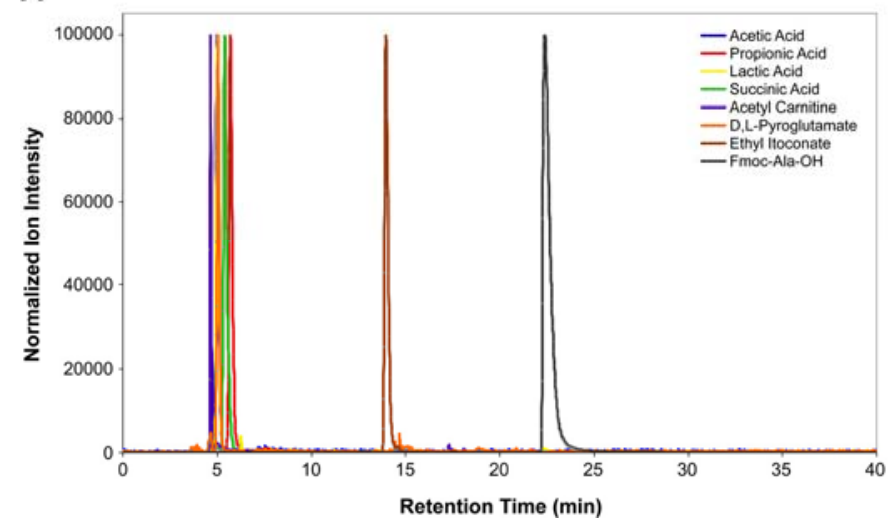

B

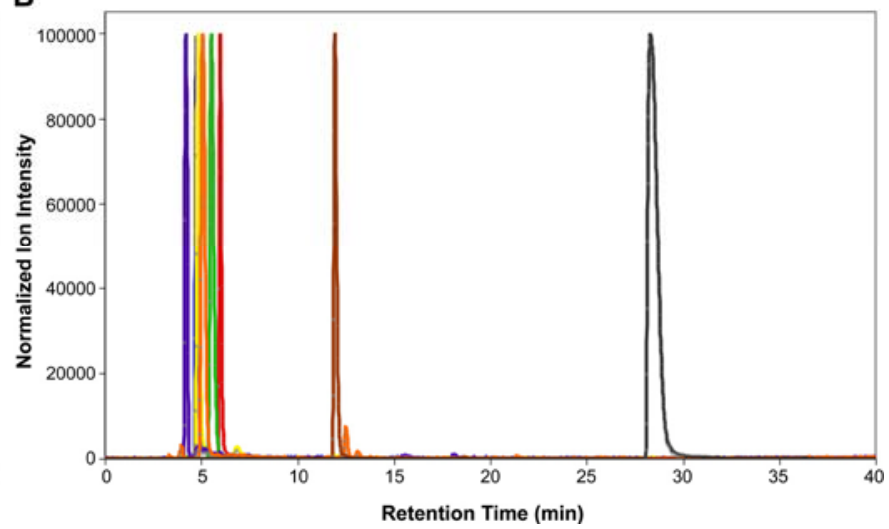

Supporting Information Figure 2. Comparison of the LC elution profiles of small-molecule standards conjugated to the original tag and resolved under the original LC elution protocol (A, derived from ref. 1) or the modified LC elution protocol used for Cl-Phe tags $(\mathbf{B})$. Note that the six polar small-molecules were poorly retained and resolved by the original tag under either LC elution protocol. Original LC elution protocol: isocratic elution of $100 \% \mathrm{~A}$ at $0.25 \mathrm{~mL} / \mathrm{min}$ for $5 \mathrm{~min}$ followed by a linear gradient of $0-100 \% \mathrm{~B}$ at $0.5 \mathrm{~mL} / \mathrm{min}$ over $35 \mathrm{~min}$, then an isocratic elution at $100 \% \mathrm{~B}$ for 9 min and re-equilibration with $100 \%$ A for 4 min (A: 95:5 $\mathrm{H}_{2} \mathrm{O}: \mathrm{CH}_{3} \mathrm{CN}, 0.1 \%$ formic acid; B: 95:5 $\mathrm{CH}_{3} \mathrm{CN}: \mathrm{H}_{2} \mathrm{O}, 0.1 \%$ formic acid). Elution times: Acetyl carnitine $t_{R}=4.66$ min, Acetic acid $t_{R}=4.98$ min, Lactic acid $t_{R}=5.00$ min, D,L-Pyroglutamate $t_{R}=$ 5.06 min, Succinic acid $t_{R}=5.39$ min, Propionic acid $t_{R}=5.70$ min. LC elution protocol for Cl-Phe tags: isocratic elution of $100 \% \mathrm{~A}$ at $0.25 \mathrm{~mL} / \mathrm{min}$ for $2 \mathrm{~min}$ followed by a the following step gradients (all at 0.5 mL/min): 0-10\% B over 3 min, 10-15\% B over 5 min, 15-35\% B over 15 min, 35-75\% B over 10 min, 75$100 \%$ B over 5 min, then an isocratic elution at 100\% B for 5 min and re-equilibration with $100 \%$ A for 4 min (A: 95:5 $\mathrm{H}_{2} \mathrm{O}: \mathrm{CH}_{3} \mathrm{CN}, 0.1 \%$ formic acid; B: 95:5 $\mathrm{CH}_{3} \mathrm{CN}: \mathrm{H}_{2} \mathrm{O}, 0.1 \%$ formic acid).. Elution Times: Acetyl carnitine $t_{R}=4.20 \mathrm{~min}$, Acetic acid $t_{R}=4.72 \mathrm{~min}$, Lactic acid $t_{R}=4.79 \mathrm{~min}, \mathrm{D}, \mathrm{L}-\mathrm{Pyroglutamate} \mathrm{t}_{\mathrm{R}}=$ 5.05 min, Succinic acid $t_{R}=5.51$ min, Propionic acid $t_{R}=5.89$ min. In contrast, as shown in Figure 2B of the manuscript, the retention and resolution of these polar compounds were markedly improved upon conjugation to the Cl-Phe tag. Acetyl carnitine $t_{R}=11.83$ min, Acetic acid $t_{R}=15.34$ min, Lactic acid $t_{R}=$ 15.54 min, D,L-Pyroglutamate $t_{R}=15.05$ min, Succinic acid $t_{R}=15.66 \mathrm{~min}$, Propionic acid $t_{R}=17.60$ min. 

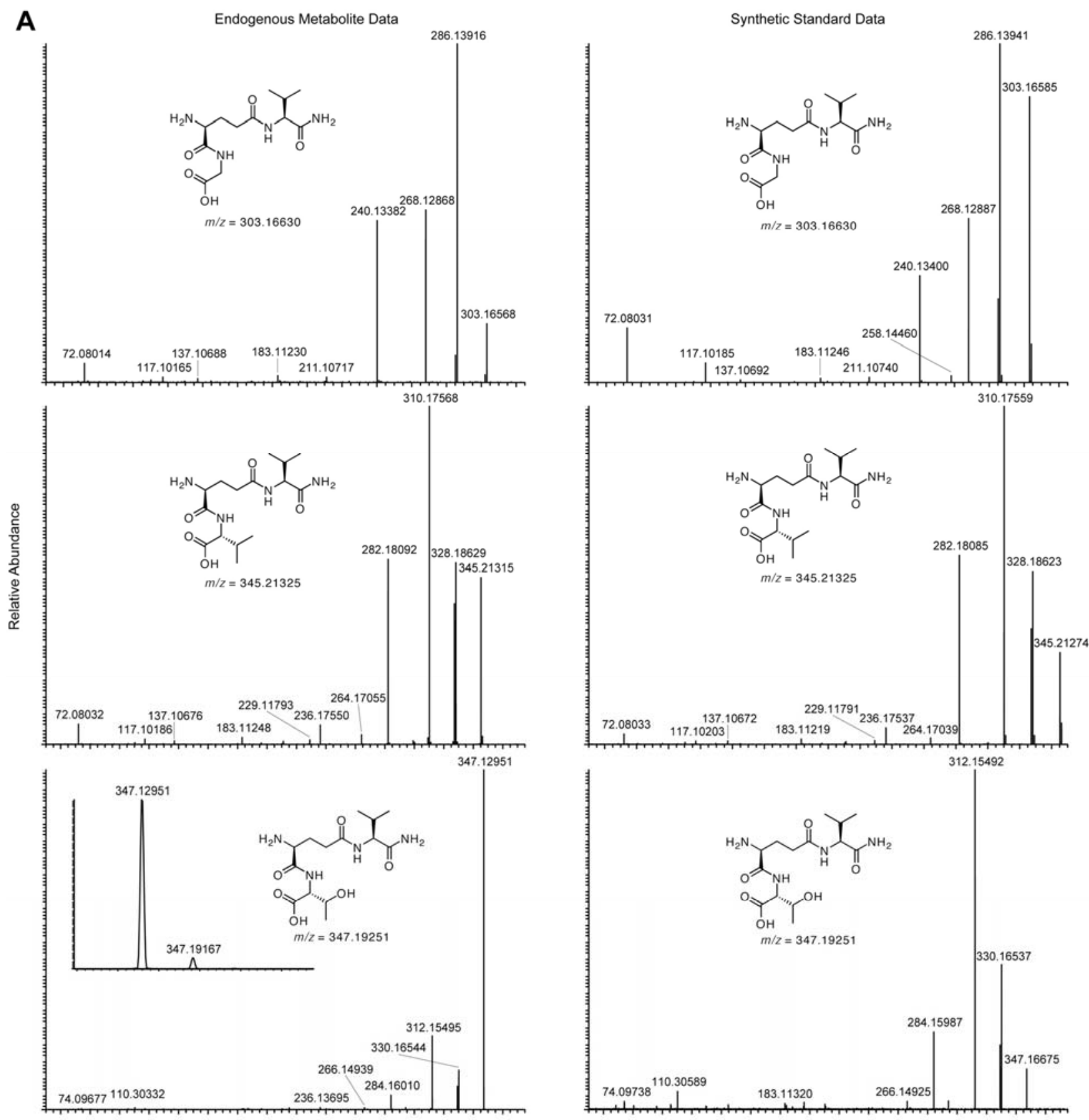


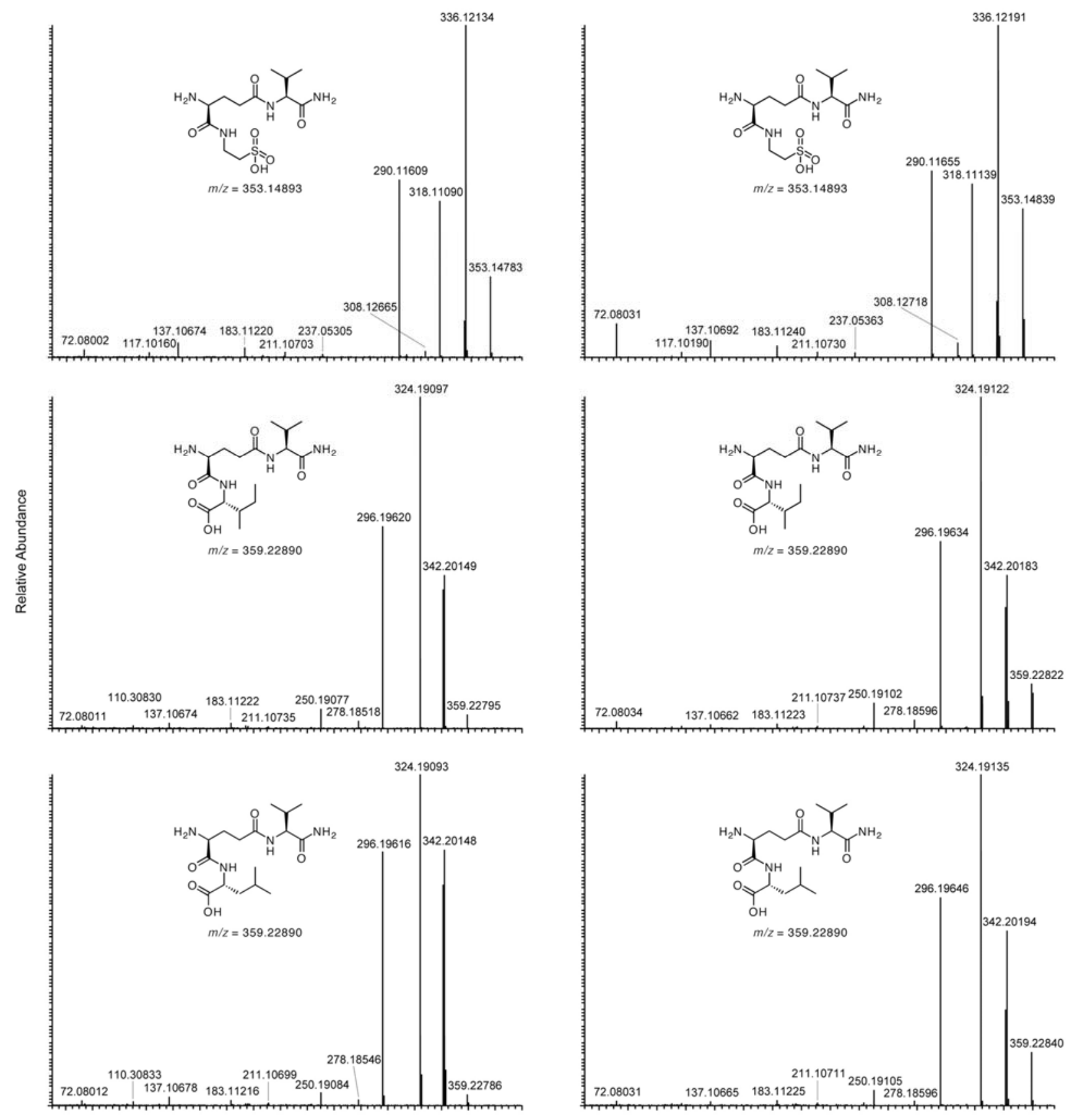




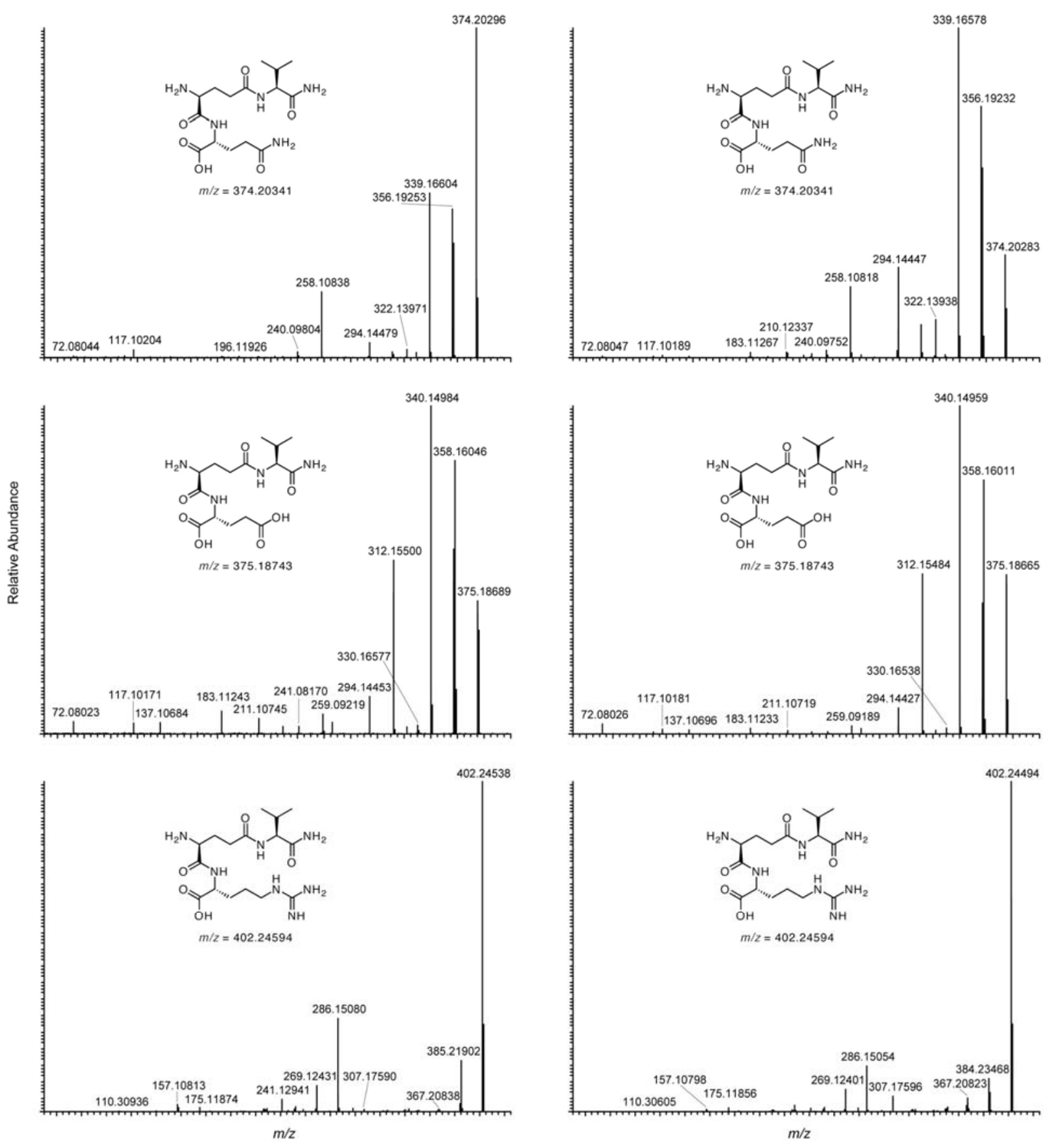


B
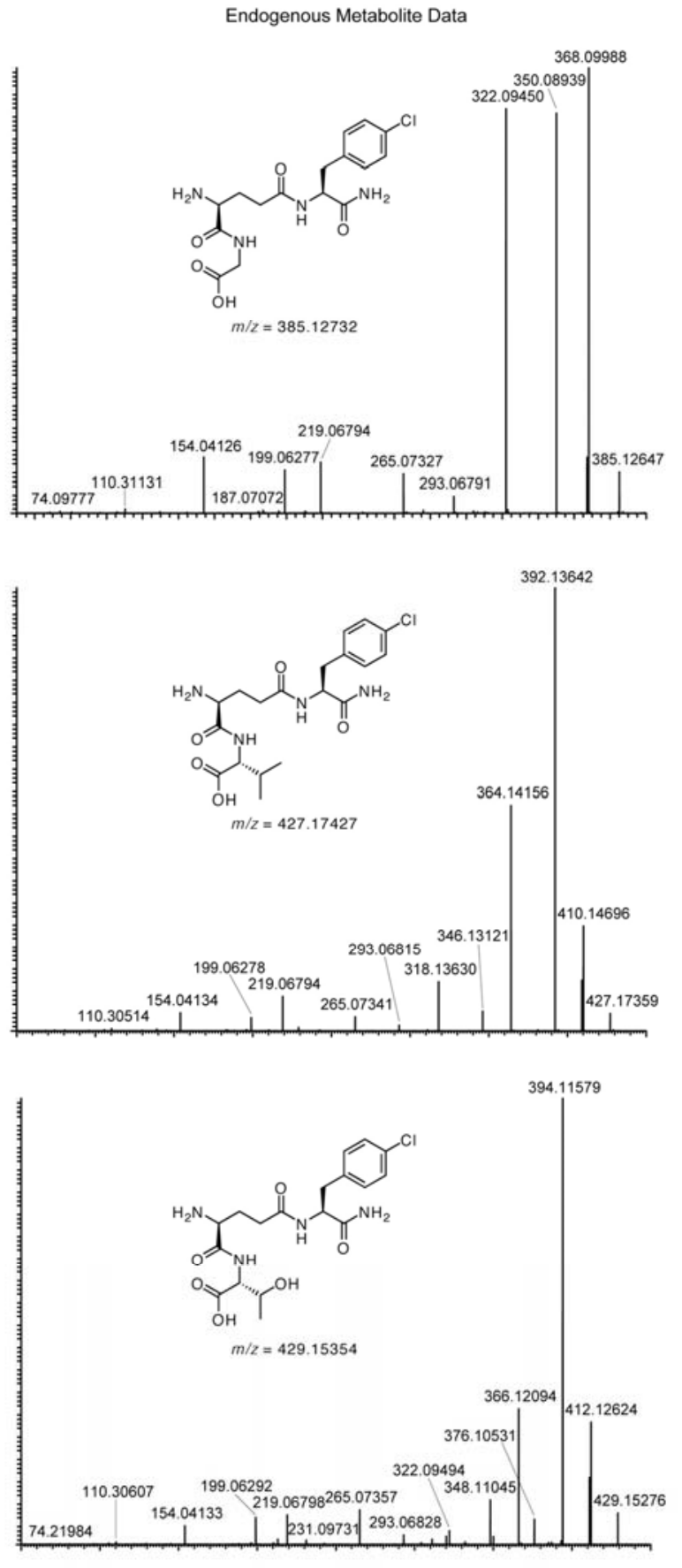

Synthetic Standard Data
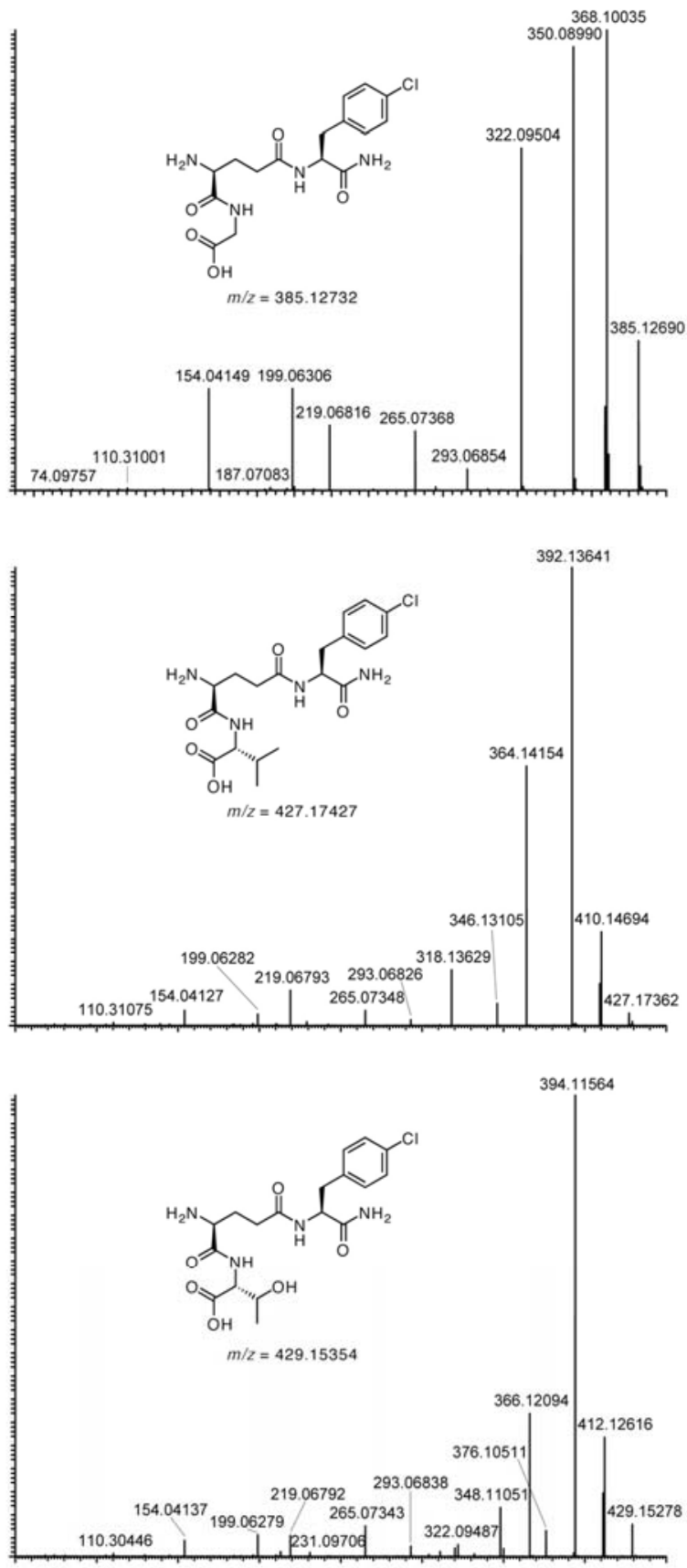

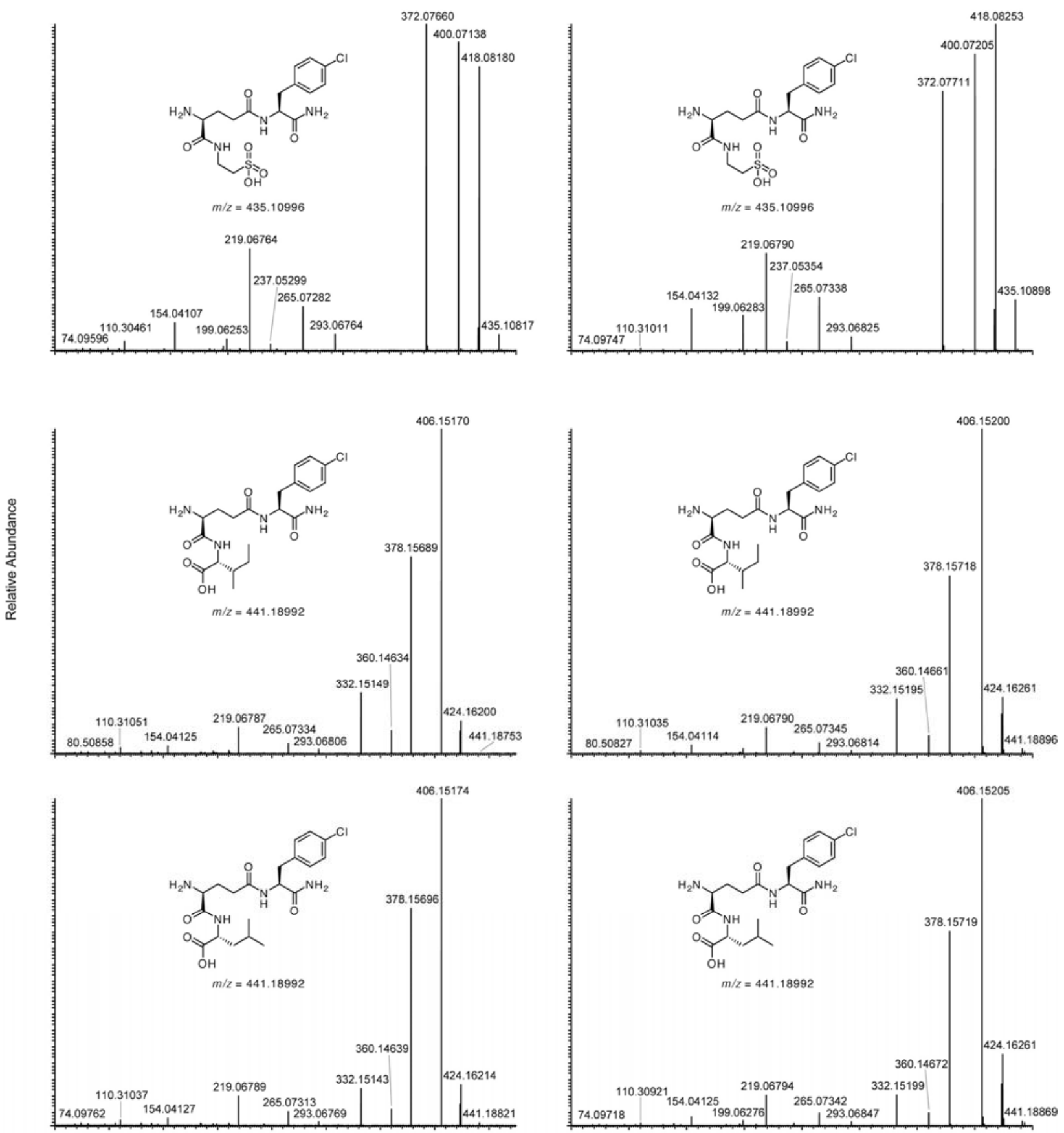

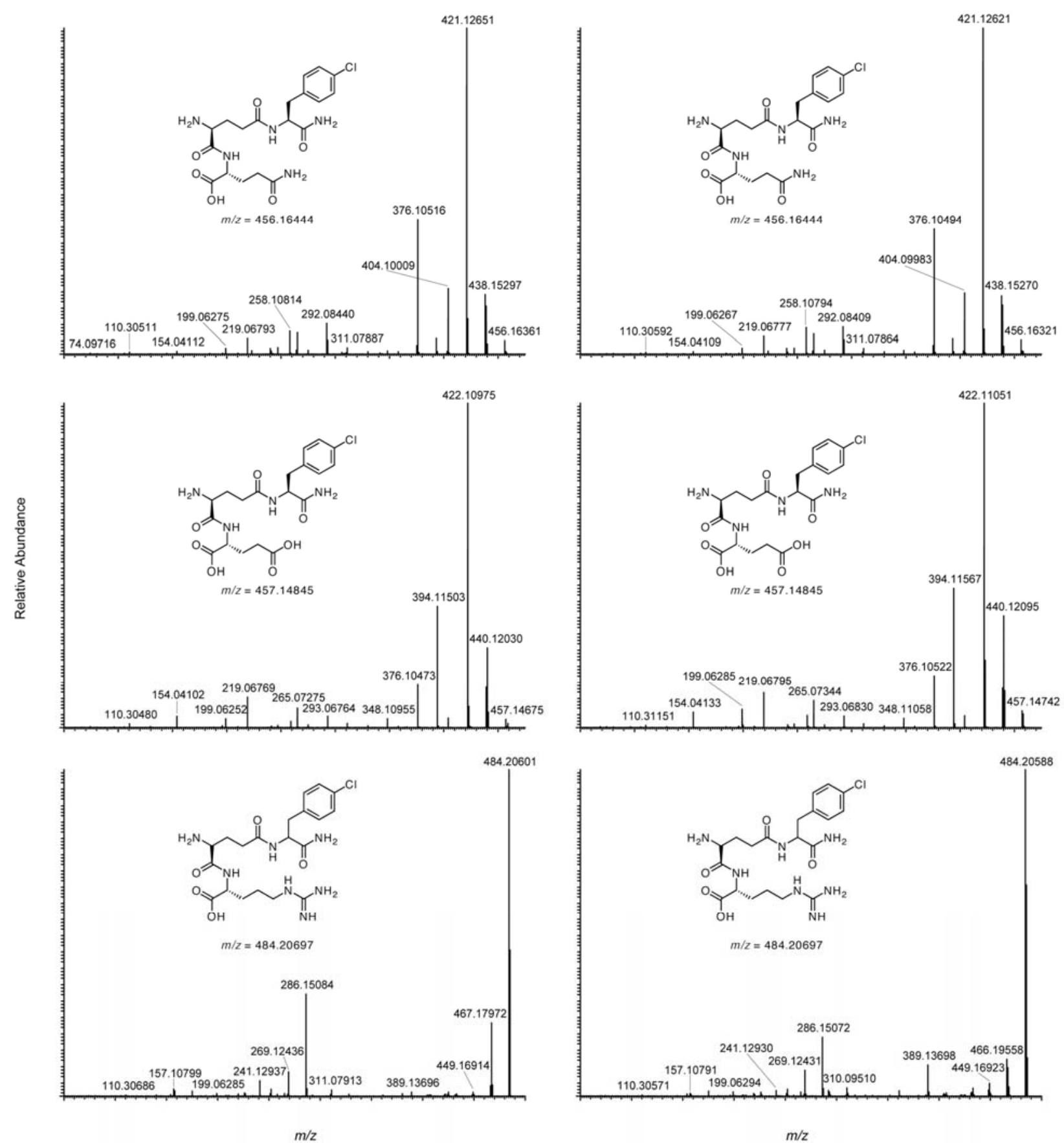

Supporting Information Figure 3. $\mathrm{MS}^{2}$ analysis of endogenous metabolites and synthetic standards with the amine-capture tags (A, original tag; $\mathbf{B}$, Phe-Cl tag). Note that when threonine was conjugated to the original tag, we were unable to resolve the parent species from a compound with a similar $\mathrm{m} / \mathrm{z}$ value (parent ion region expanded in insert). Isoleucine and leucine could not be differentiated by their fragmentation pattern. Co-elution studies confirmed the identity of the two species. 
A
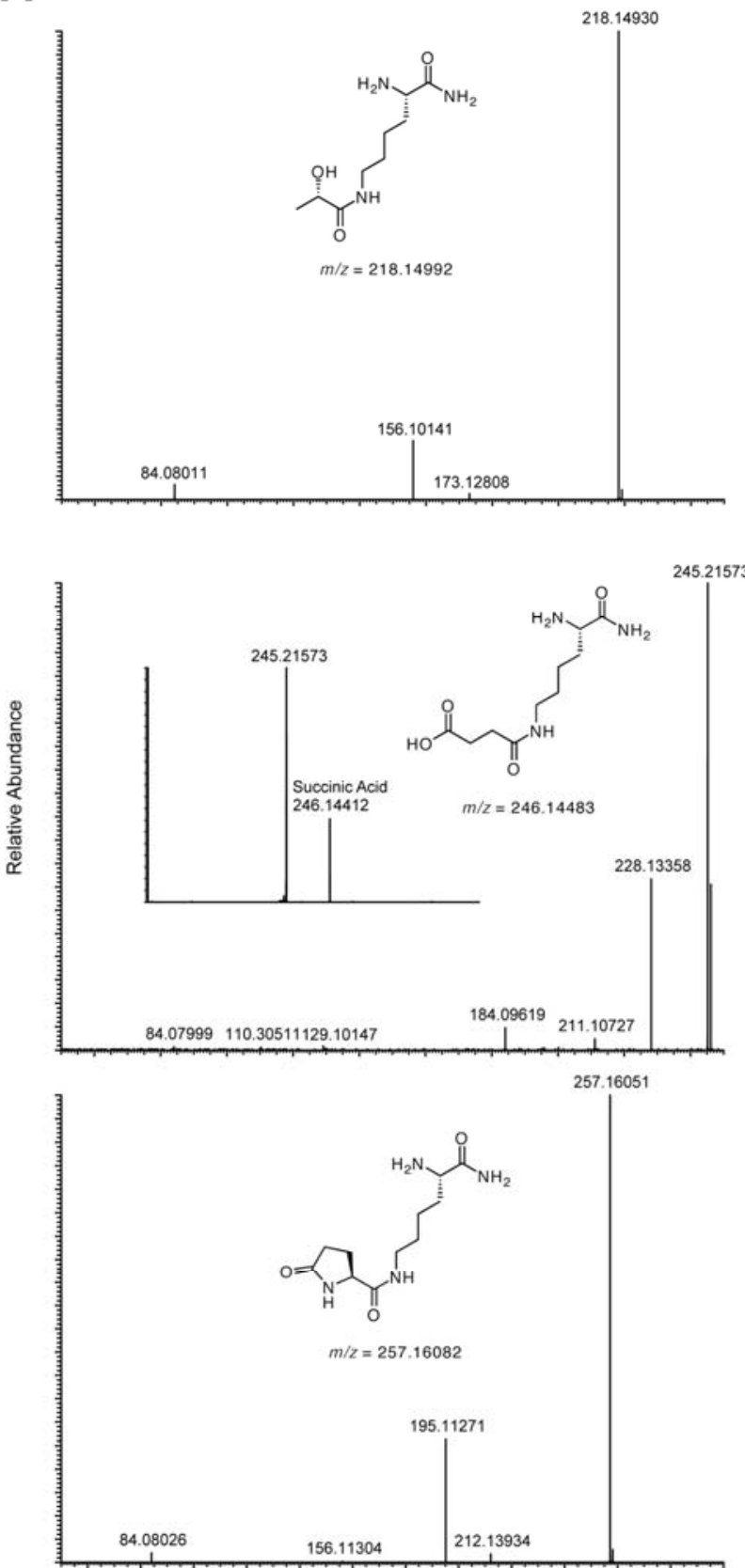

Synthetic Standard Data

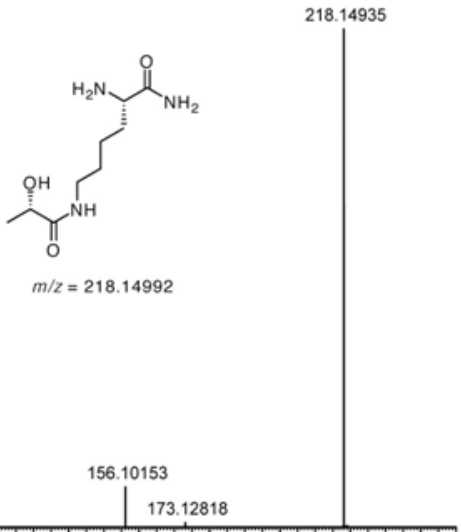

84.08029

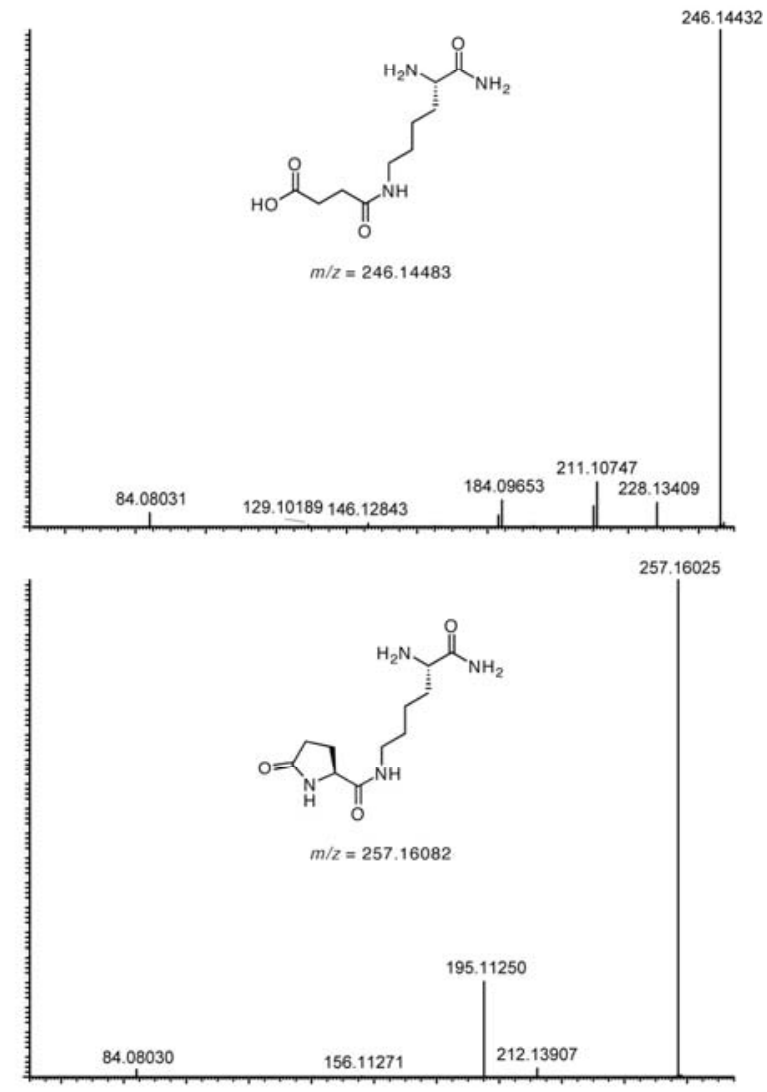



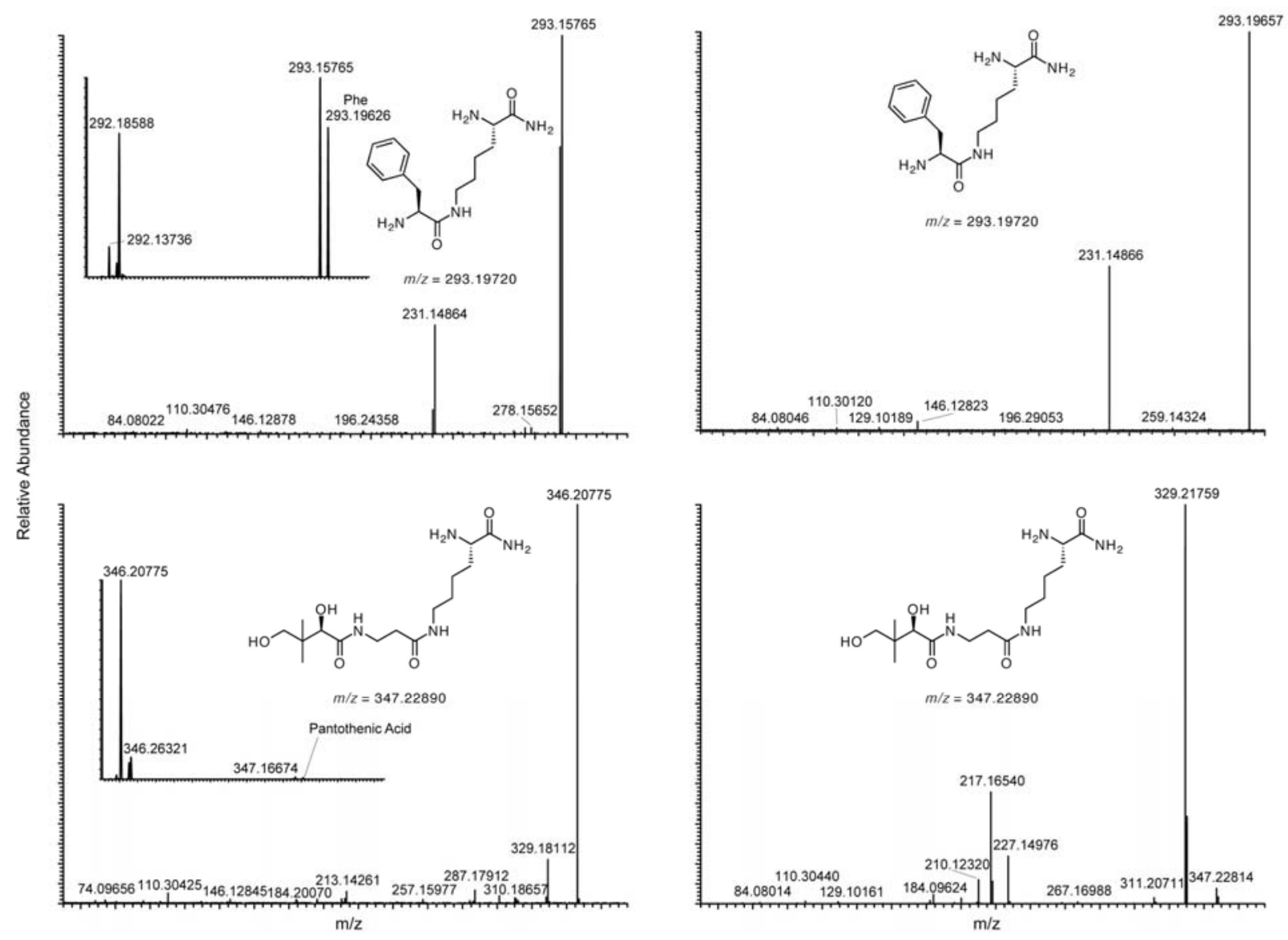
B

Endogenous Metabolite Data
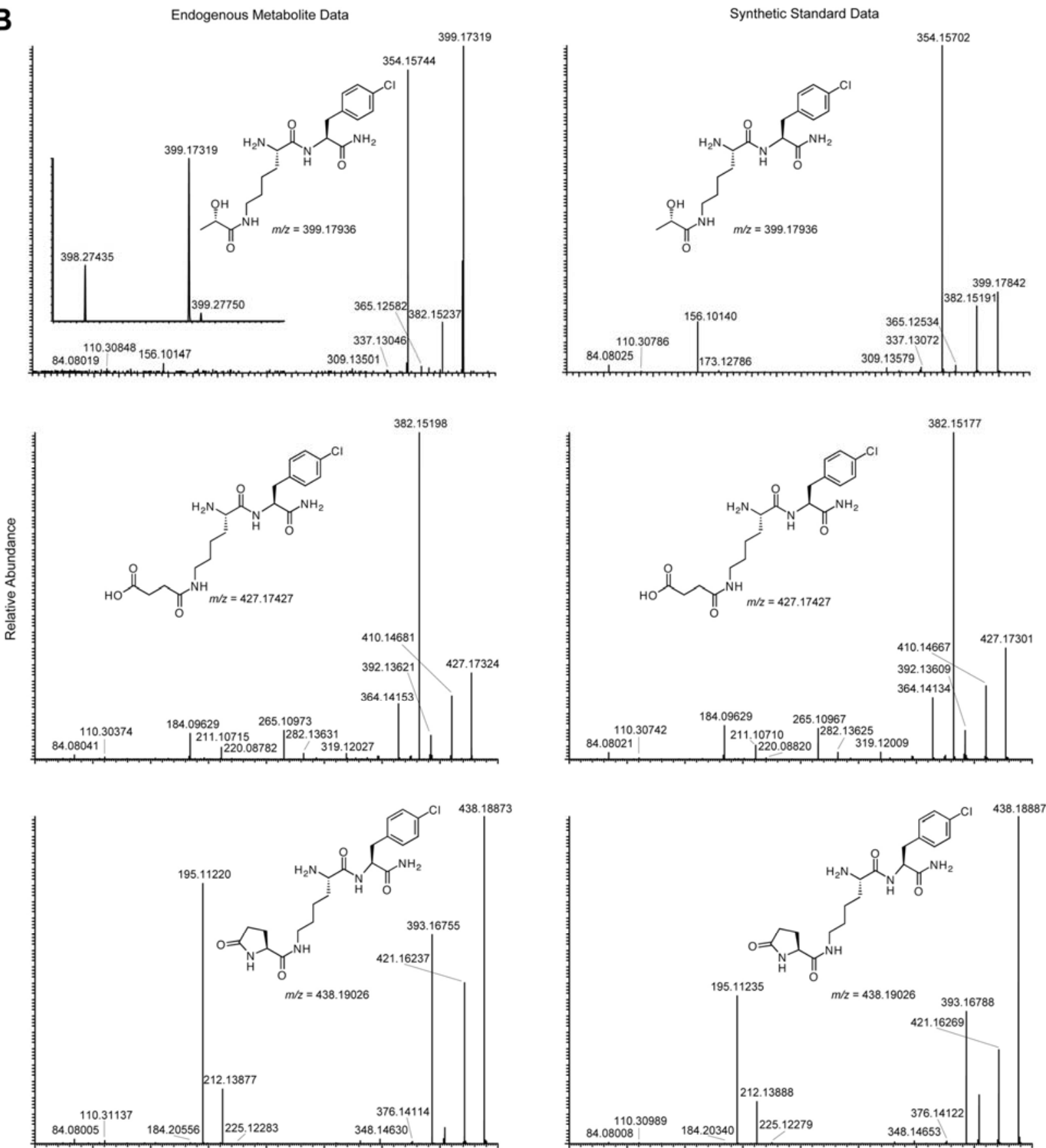

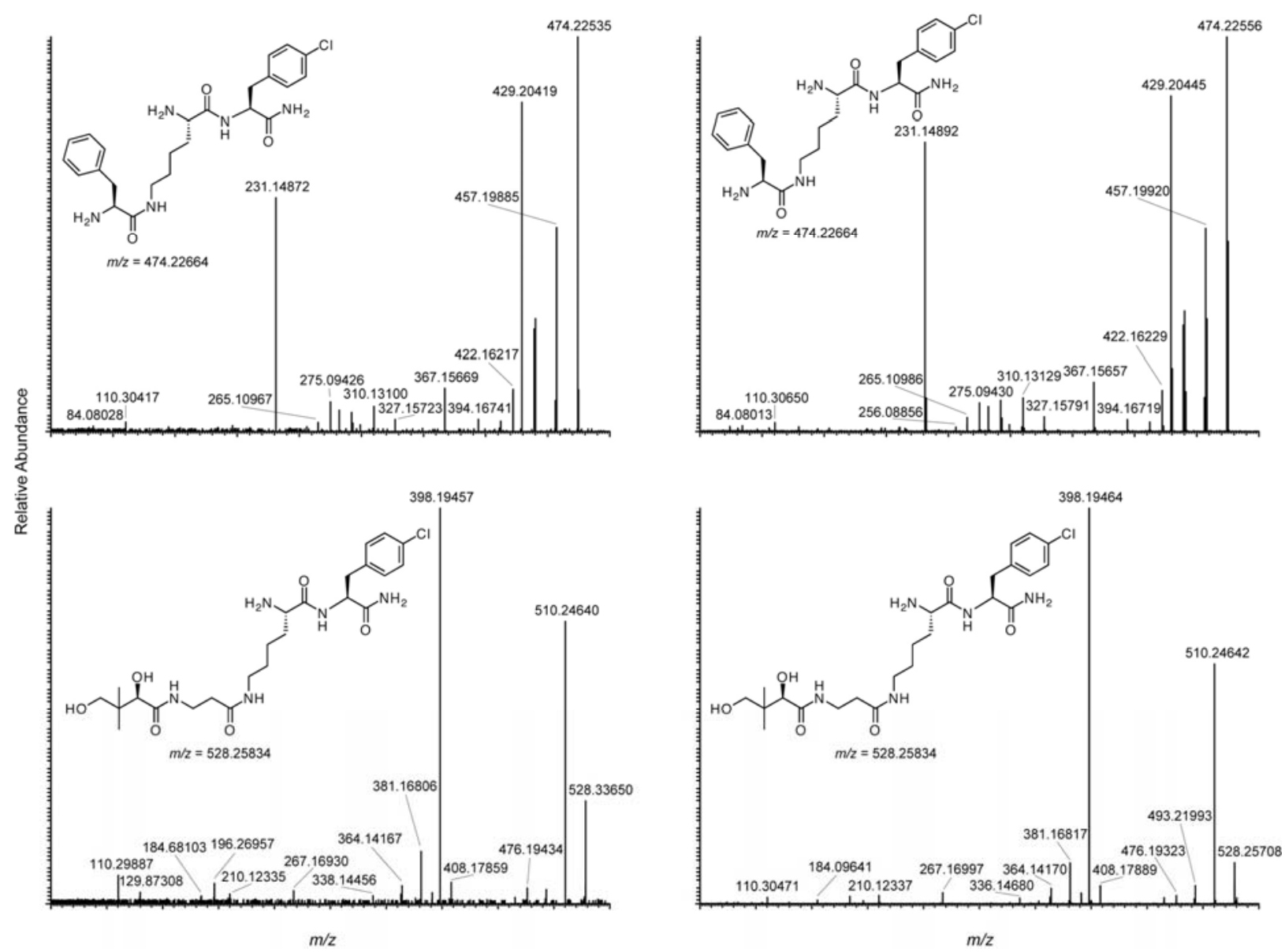

Supporting Information Figure 4. $\mathrm{MS}^{2}$ analysis of endogenous metabolites and synthetic standards with the acid-capture tags (A, original tag; $\mathbf{B}$, Phe-Cl tag). We were unable to resolve several of the metabolites (i.e., succinic acid, phenylalanine, pantothenic acid with original tag; lactic acid with Cl-Phe tag) from compounds with similar $\mathrm{m} / \mathrm{z}$ values (parent ion regions expanded in inserts). 


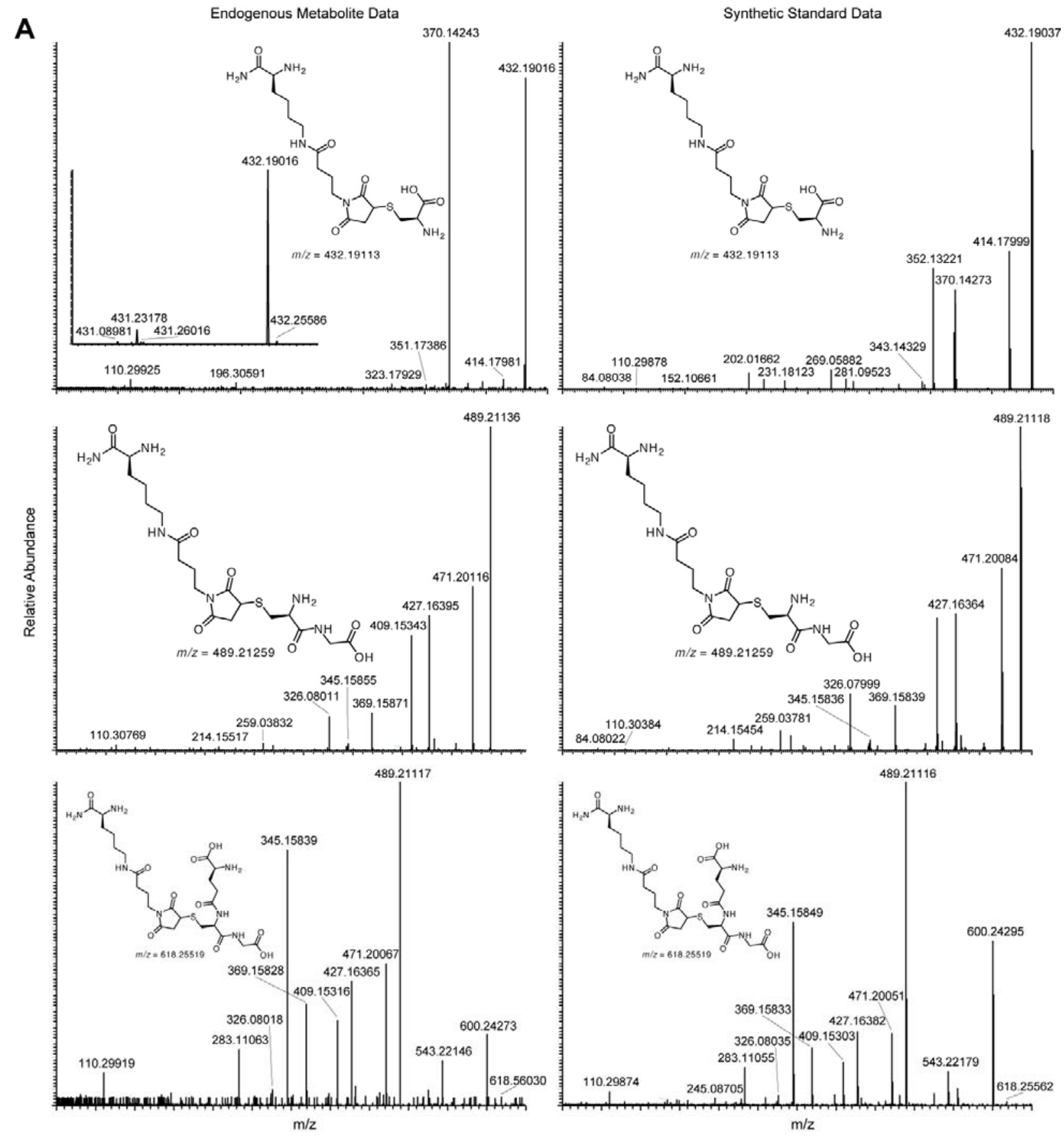


B
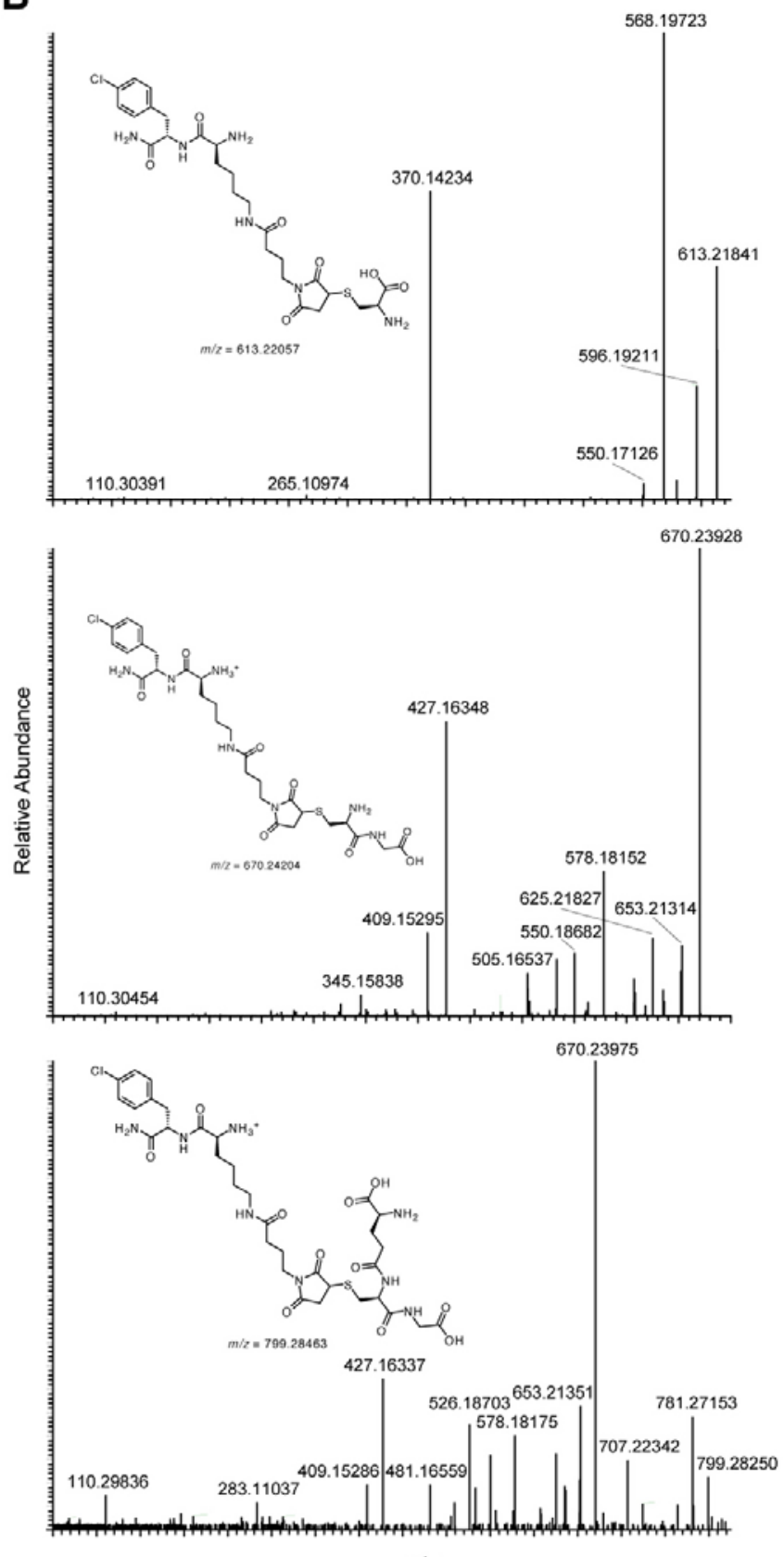

$\mathrm{m} / \mathrm{z}$
Synthetic Standard Data
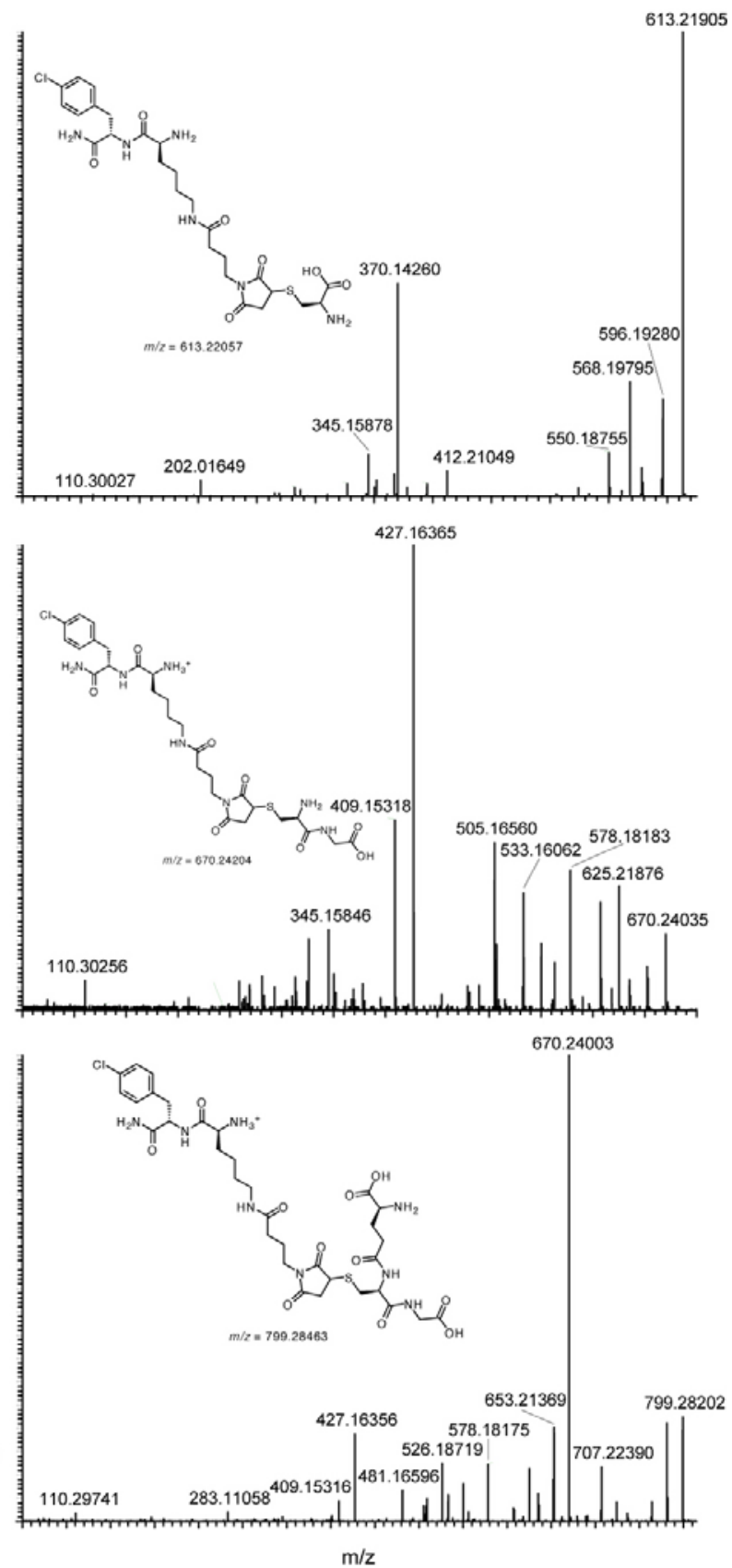

Supporting Information Figure 5. MS $^{2}$ analysis of endogenous metabolites and synthetic standards with the thiol-capture tags (A, original tag; $\mathbf{B}$, Phe-Cl tag). Note that when cysteine was conjugated to the original tag, we were unable to resolve the parent species from a compound with a similar $m / z$ value (parent ion region expanded in insert). 

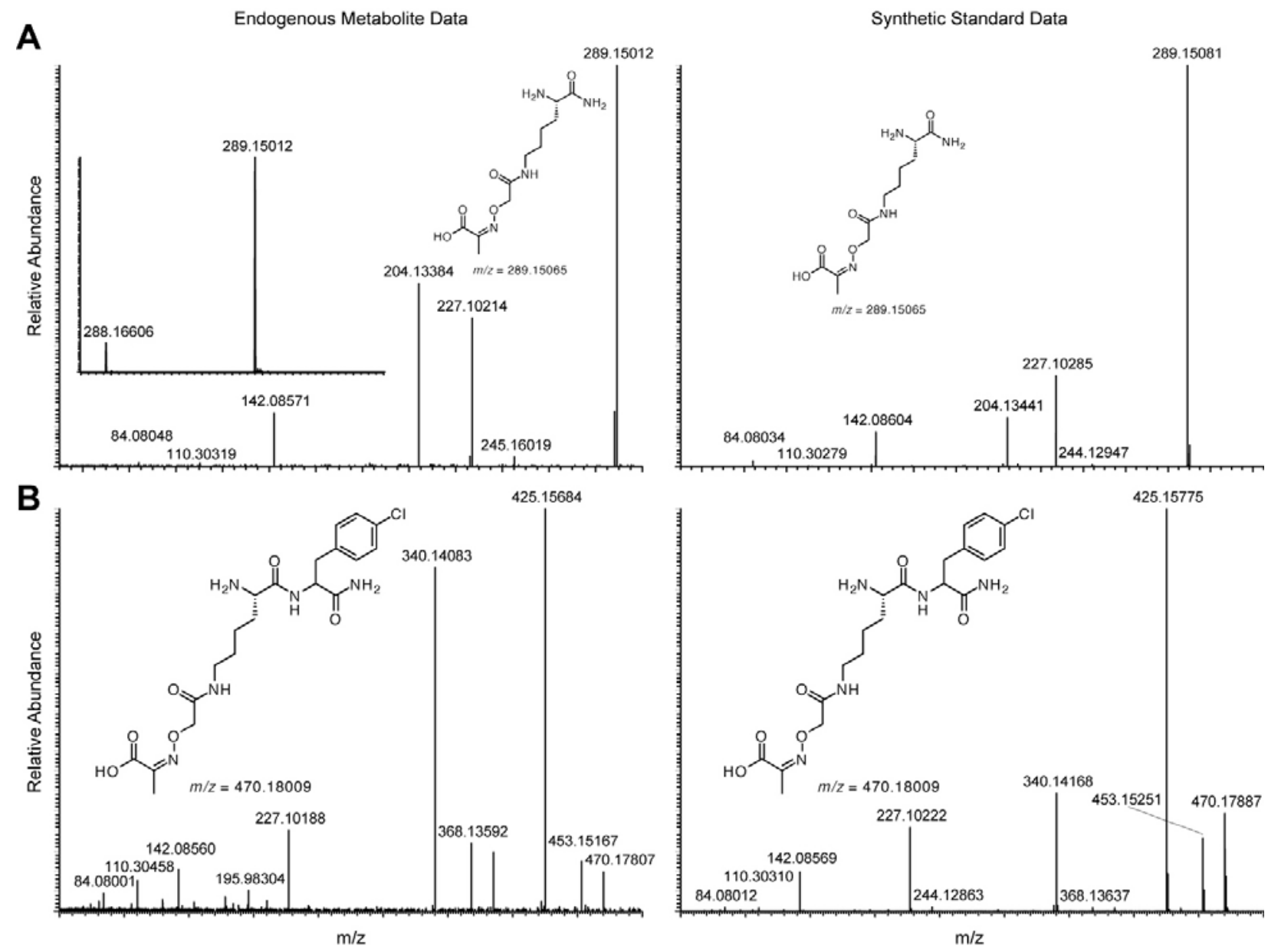

Supporting Information Figure 6. $\mathrm{MS}^{2}$ analysis of endogenous metabolites and synthetic standards with the ketone/aldehyde-capture tags (A, original tag; $\mathbf{B}$, Phe-Cl tag). Note that when pyruvate was conjugated to the original tag, we were unable to resolve the parent species from a compound with a similar $\mathrm{m} / \mathrm{z}$ value (parent ion region expanded in insert). 

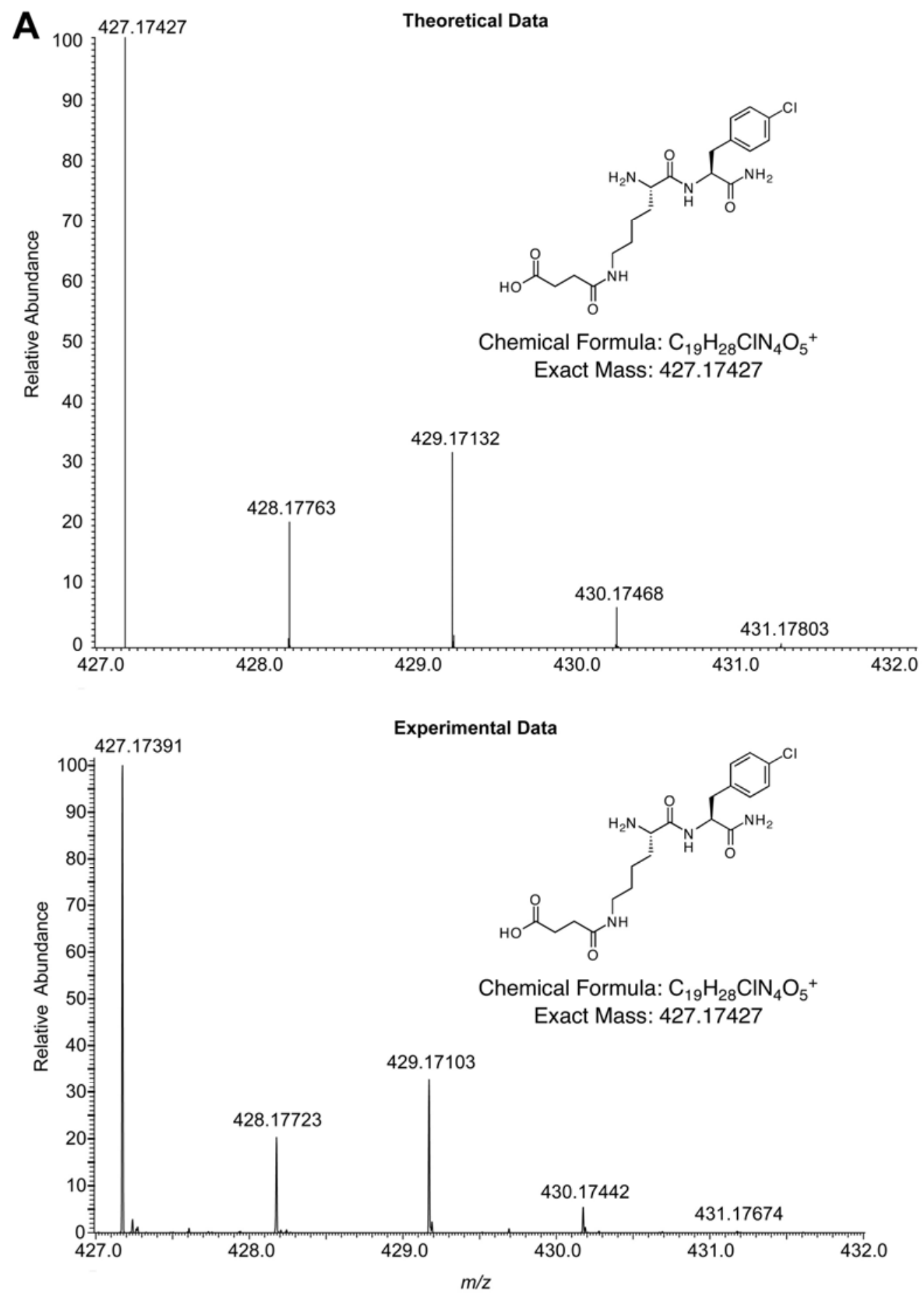

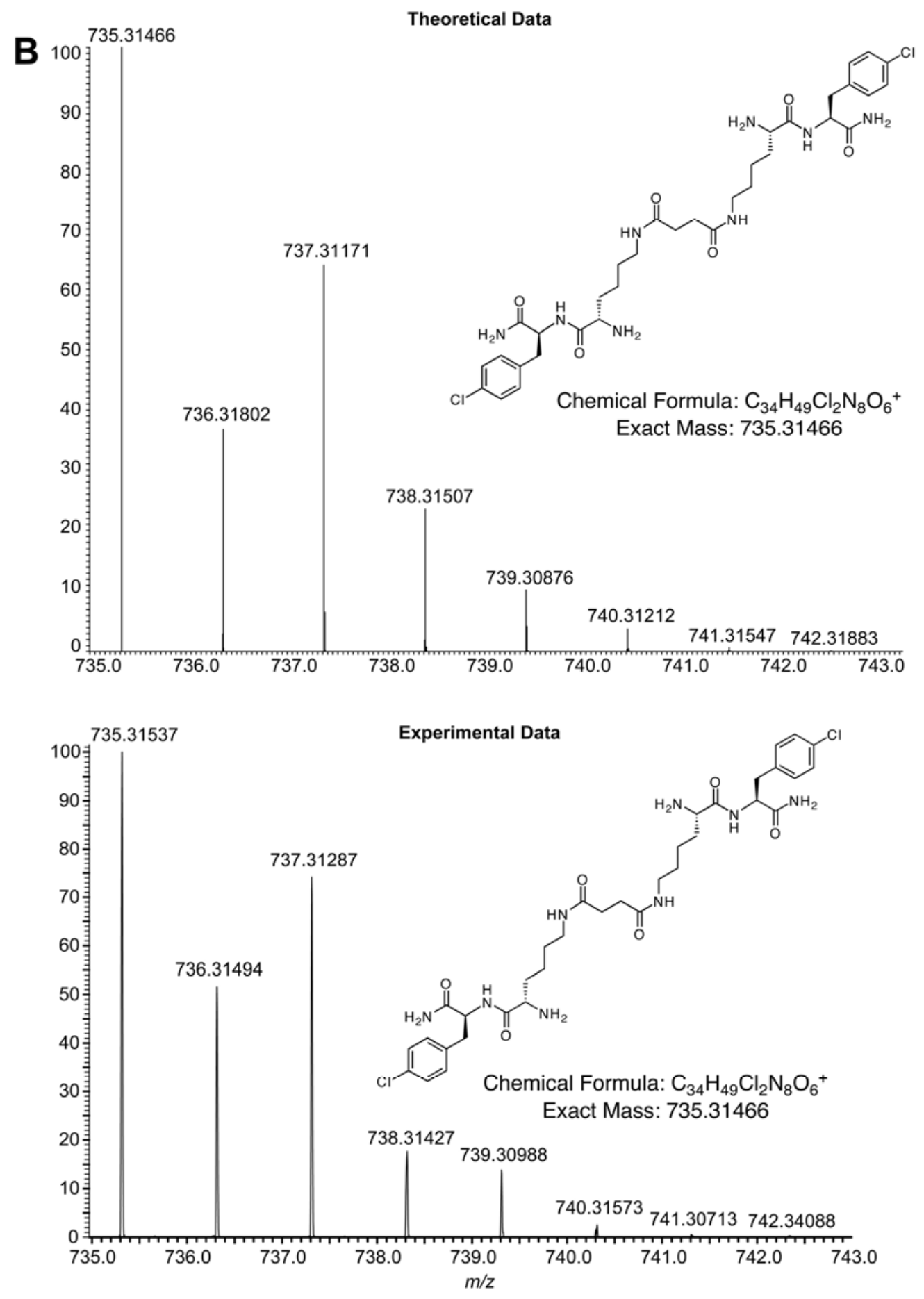

Supporting Information Figure 7. Theoretical (Top) and experimental (Bottom) isotopic abundance patterns for singly- (A) and multiply-labeled (B) succinic acid. Theoretical data generated by Xcalibur ${ }^{\mathrm{TM}}$ software.

\section{References}


1. Carlson, E.E.; Cravatt, B. F. Nat. Methods 2007, 4, 429-435.

2. http://www.thermo.com/com/cda/product/detail/1,,10123241,00.html?ca=pqd 